at seizure onset, a diffuse irritative zone on EEG, and extensive focal cortical resections affecting multiple lobes. Patients having hemispherectomies did better than those with focal cortical resections. (Sugimoto T, Otsubo H, Hwang PA, Hoffman HJ, Jay V, Snead OC III. Outcome of epilepsy surgery in the first three years of life. Epilepsia May 1999;40:560-565). (Reprints: Dr T Sugimoto, Department of Pediatrics, Kansai Medical University Otokoyama Hospital, Izumi 19, Otokoyama, Yawata, Kyoto, 614 Japan).

COMMENT. Young children with refractory epilepsy may benefit from surgery, especially in those showing concordance of ictal video-EEG and neuroimaging data. The outcome in children undergoing hemispherectomy is superior to results of focal cortical resection.

\title{
EARLY-ONSET BENIGN OCCIPITAL SEIZURE SYNDROME
}

The recognition of a syndrome of early-onset benign childhood occipital seizures (EBOS) is proposed in a report from St Thomas' Hospital. London, England. The characteristic findings are infrequent partial, usually nocturnal, seizures with deviation of the eyes and vomiting, frequently evolving to hemi- or generalized convulsions, with onset between 1 and 12 years, usually at age 5 years. Behavioral changes with irritability are frequent, and retching, coughing, and incontinence may occur. The prognosis is excellent, one third having only one seizure, and remission occurs within one year from onset. EEG shows occipital spikes, especially in sleep. Centrotemporal spike foci may occur later and a few develop rolandic seizures. (Panayiotopoulos CP. Early-onset benign childhood occipital seizure susceptibility syndrome: a syndrome to recognize. Epilepsia May 1999;40:621-630). (Reprints: Dr CP Panayiotopoulos, St Thomas' Hospital, London SE1 7EH, England).

COMMENT. Dr Panayiotopoulos proposes an addition to the classification of childhood epilepsy syndromes, with recognition of an early-onset benign occipital seizure syndrome (EBOS), sharing identical EEG manifestations with lateonset idiopathic occipital epilepsy (LOE), but having more common clinical features with rolandic seizures (BECTS). EBOS are infrequent, mainly nocturnal, and remit usually within one year, whereas LOE are diurnal and usually persist for years. A unified concept for benign childhood partial epilepsies might be preferred, since clinical and EEG characteristics are often shared, and one syndrome may evolve into another. We must await the determination of genetic markers.

\section{RISK OF SEIZURE-RELATED AUTOMOBILE ACCIDENTS}

A retrospective case-control study to identify clinical risk factors for seizure-related motor vehicle crashes in 50 patients with epilepsy and 50 matched control patients was performed at Johns Hopkins University, Baltimore, MD. The majority $(54 \%)$ of patients who crashed were driving illegally, having a history of poor seizure control; $25 \%$ had more than one crash related to a seizure and $20 \%$ had missed a dose of medication just prior to the crash. Patients with well controlled epilepsy, and no seizure recurrence for more than 12 months had a $93 \%$ reduction in risk of a crash, compared to patients with more frequent seizure recurrence. The presence of reliable auras with seizures, and few prior nonseizure-related accidents decreased the odds of seizure-related accidents. (Krauss GL, Krumholz A, Carter RC, Li G, Kaplan P. Risk factors for seizure-related motor vehicle crashes in patients with epilepsy. Neurology April 1999;52:13241329). (Reprints: Dr Gregory L Krauss, Meyer 2-147, 600 N Wolfe St, Baltimore, MD 21287). 
COMMENT. Patients with epilepsy of driving age may be counseled that factors known to reduce the risk of seizure-related automobile accidents include optimal AED therapy with long seizure-free intervals, reliable auras, and few prior nonseizure-related accidents. The authors comment that if the 50 patients who had crashed had honored a 12-month legally defined seizure-free restriction, $74 \%$ of crashes might have been averted. A longer seizure-free restriction on driving could lead to poor compliance and a higher risk of crashes. Patients whose medication was closely monitored and optimized showed an $89 \%$ reduction in risk of crashes.

Berg AT and Engel J Jr, in an editorial, discuss "Restricted driving for people with epilepsy." (Neurology April 1999;52:1306-1307). The minimum seizurefree interval of 12 months is the strongest predictor of avoidance of motor vehicle accidents for patients with epilepsy. This 1-year restriction on driving is generally appropriate and is mandated by the European Union Association of International Bureau for Epilepsy. A US consensus development workshop opinion, approved by the AAN, AES, and EFA, recommends a minimum 3-month seizure-free interval, recognizing the importance of the neurologist's input and need to consider mitigating factors. The study by Krauss et al provides evidence for some of the factors that may permit reduction of the 1-year restriction in certain individuals: eg reliable aura, accident-free prior driving record, and close AED monitoring and compliance.

\section{HEREDO-DEGENERATIVE DISEASE}

\section{INFANTILE NEUROAXONAL DYSTROPHY: DIAGNOSTIC CRITERIA}

The diagnostic significance of clinical, neurophysiologic, and neuroradiologic findings in 13 patients with infantile neuroaxonal dystrophy (INAD) was evaluated at the National Neurological Institute, Milan, Italy. Symptoms and signs of psychomotor regression presented between 6 months and 2 years of age. The clinical course was typical in 9 patients, with rapid motor and mental deterioration: it was atypical in 4 showing a slower rate of progression. Late neurologic features included optic atrophy and vision failure in 8 , nystagmus in 3 , and spastic or areflexic tetraparesis with axial hypotonia and distal contractures in all patients. EMG signs of chronic denervation, EEG fast activity, and abnormal visual evoked potentials occurred in all patients during the course of the disease. NCS became abnormal and consistent with axonal sensorimotor peripheral neuropathy in 9. All patients had histologic evidence of spheroids on skin biopsy. Characteristic MRI abnormalities were cerebellar atrophy, first involving the inferior vermis and later the cortex, with hyperintense signal on T2-weighted images, and hypointensity in the pallida and substantia nigra. a-N-acetyl-galactosaminidase leukocyte activity was normal in 10 patients tested. (Nardocci N, Zorzi G, Farina L et al. Infantile neuroaxonal dystrophy. Clinical spectrum and diagnostic criteria. Neurology April 1999;52:1472-1478). (Reprints: Dr Nardo Nardocci, Department of Child Neurology, National Neurological Institute "Carlo Besta," Via Celoria 11, 20133 Milan, Italy).

COMMENT. The diagnostic criteria for INAD are as follows: 1) spheroids on skin biopsy; 2) onset before 3 years; 3) psychomotor deterioration, with symmetric pyramidal tract signs and truncal hypotonia; and 4) progression to spastic tetraparesis, blindness, and dementia by the age of 4 years. Atypical cases may occur, with slower progression beginning between 7 and 12 years after a stable course resembling static encephalopathy, and hypotonic-areflexic 